\title{
Effect of Female Genital Mutilation on Sexual Relation among Married Women at Tanta City
}

\author{
Manal Abdalla Sayed Ahmed Gaheen ${ }^{1}$, Azza Fouad Mohamed El-Adham². \\ Lecturer of Maternity and Gynecological Nursing Dept., Faculty of Nursing, Tanta University. Lecturer of \\ Maternity and Gynecological Nursing Dept., Faculty of Nursing, Tanta University.
}

\begin{abstract}
Background: Female genital mutilation (FGM) is a traditional dangerous abusive practice to human rights of females at different age groups worldwide. It is a socio-cultural, legal and health issue affects the lives and sexual health of females. The aim of the present study was to investigate the effect of FGM on sexual relation among married women. An exploratory descriptive design was used to collect the data from family planning clinics of both Tanta University, and El-Menshawy Hospitals at Tanta City. The study included a convenient sample of 80 women attending the previously mentioned settings. They were equally divided into 2 groups. The first group consisted of 40 circumcised women, 20 women from each hospital. The second group consisted of 40 non-circumcised women, 20 women from each hospital. Three tools were used in this study. Tool I: An interview questionnaire included the socio - demographic characteristics of the women. Tool II: Clinical observation sheet to confirm the degree of female genital cutting and the presence of external female genitalia deformity. Tool III: A modified Arizona Sexual Experiences Scale (ASEX) for assessment of women sexual dysfunction. The results showed that sexual dysfunction was significantly higher among circumcised women compared to non-circumcised ones $(97.5 \%$ \& $85 \%)$ respectively $(P=0.038 *)$. As regard the types of FGM, nearly one third of circumcised women had female genital mutilation type I and more than two- thirds of them had type II. It also shows that $88.89 \%$ of circumcised women who have partial clitoridectomy and $100 \%$ of them who have complete clitoridectomy were suffering from sexual dysfunction. Conclusion, the study concluded that there was a highly significant difference between circumcised and non-circumcised women and sexual relation. This study recommended that there is a crucial need for focusing on more orientation of the community regarding the effect and the hazard of FGM on sexual relation and its role on occurrence of sexual dysfunction. Keywords: Female genital Mutilation, female genital cutting, female circumcision, sexual relation,sexual function and sexual dysfunction.
\end{abstract}

\section{Introduction}

Female genital mutilation (FGM) or female genital cutting (FGC) is a harmful traditional practice, and is a violation of the human rights of women worldwide. It is often associated with physical, psychological, sexual and social consequence. FGM is defined by the World Health Organization (WHO), United Nations Children's Fund, and the United Nations Population Fund, as partial or total removal of the female external genitalia or other injury to the female genital organs for cultural or other non-therapeutic reasons ${ }^{(1,2)}$. The WHO classified FGM into four types as follows: type 1 consists of excision of the prepuce, with or without excision of clitoris, type II involves clitoridectomy and partial or total excision of labia minora, type III includes removing part or all of the external genitalia and re-approximation of the remnant labia majora leaving a small neointroitus and type IV involves other forms of injuries to the genital region including pricking, piercing, stretching, burning, scraping or any other manipulation of external genitalia ${ }^{(3,4)}$.

The WHO estimated that more than 200 million girls and women alive today have undergone female genital mutilation in the countries where the practice is concentrated. Furthermore, there are an estimated 3 million girls at risk of undergoing female genital mutilation every year ${ }^{(4)}$. United Nations Children's Emergency Fund( UNICEF) reported in November 2016 that prevalence rates for sub-Saharan Africa were $39 \%$ for women and $17 \%$ for girls aged 0-14; for Eastern and Southern Africa 44 and $14 \%$, and for West and Central Africa 31 and $17 \%{ }^{(5)}$ As of 2013, in Egypt, Ethiopia and Nigeria the number of women and girls living with FGM had the highest percentage (27.2 million, 23.8 million and 19.9 million) respectively. ${ }^{(6)}$ Compared to $96 \%$ of women in Egypt had undergone some form of genital cutting at 2007 , which mean that the rate declined, but there is still a need for a clinical strategy for completely eradicate this bad traditional cultural practice to prevent its consequences ${ }^{(7)}$. 
FGM is still widely performed by non-professionals, instead of the efforts to prevent hospitals and medical doctors from carrying out the procedure. Recently, a 12-year-old Egyptian girl died while undergoing the procedure $^{(7)}$. The WHO reported that Female genital cutting is a deeply-rooted traditional practice in more than 28 countries in Africa includes Egypt, plus some countries in the Middle East and Asia ${ }^{(4)}$. The importance given to virginity and an intact hymen in the Egyptian society is the reason why female genital cutting remains a very widespread practice. Traditional beliefs of removing parts of girls' external genitals organs, minimized sexual desire and permits a female who has reached the dangerous age of puberty and adolescence to protect her virginity and therefore her honor, with greater ease ${ }^{(8)}$. FGM enhances the sexual pleasure of men while genitally mutilated women sense little or no sexual pleasure. This denial of sexual pleasure can have psychological effects on women, such as post traumatic stress disorder (PTSD). These women therefore become sexual objects and reproductive vehicles for men ${ }^{(9)}$. Islam's teachings prohibit inflicting harm on any human being. Thus, FGC is contrary to Islam, an attack on women, and a practice that should be criminalized ${ }^{(10,11)}$.

The WHO states that all types of female genital cutting involve removal or damage to the normal external female genitalia can lead to a range of physical, sexual, and psychological complications ${ }^{(12)}$. The area of the clitoris and labia has a dense concentration of nerves and are highly sensitive. Because most procedures are done without anesthesia, most women experience extreme pain. Applying local anesthesia to the vulva is in itself extremely painful. Primary hemorrhage represents $25 \%$ of all reported complications of FGC as a result of amputation of the clitoris involving the clitoral artery in which blood flows at high pressure. Secondary hemorrhage can occur after several days with sloughing of the clot on the clitoral artery due to infection ${ }^{(12)}$. Infection is caused by follow septic technique and contamination of the wound by feces, urine, or blood. Infection can range from a superficial infection to abscesses, septicemia and gangrene of vulvar tissue ${ }^{(2) .}$ Shock can occur from hemorrhage, pain, psychological trauma, or sepsis. If the urethral meatus becomes injured, it may develop a meatal obstruction. The evidence on obstetric and gynecological complications is mixed: increased risks have been reported for some complications of labor and delivery such as, hemorrhage, infection, urine retention, obstructed labor, shock, and death ${ }^{(13,14)}$.

Other complications of FGM include scarring complications, such as keloids, dermoid cysts, introital and vaginal stenosis. These lead to dyspareunia, women's sexual dysfunctions include persistent or recurrent disorders of sexual interest/desire, disorders of subjective and genital arousal, orgasm disorder, pain and vaginismus, and difficulty with attempted or completed intercourse ${ }^{(15-17)}$. Every woman has the right to have sexual health and to feel sexual pleasure for full psychophysical well-being of the person. Many studies underscore the difficulties of research on the health and sexuality consequences of FGM ${ }^{(17) . ~ O t h e r ~ s t u d i e s ~}$ emphasized that understanding the complications and consequences of female genital mutilation/ cutting is necessary to prevent the continuation of such practice that is associated with human rights violations. Additionally, many health care professionals in different countries of the world lack experience in assisting women with female genital mutilation during pregnancy and birth, and they are usually untrained in this aspect of care. Thus, the present study aimed to investigate the effect of female genital mutilation on sexual relation.

\section{Aim of the study}

To investigate the effect of female genital Mutilation on sexual relation among married women in Tanta City . Research Hypothesis:

Women with FGM experience more sexual dysfunction than women without.

Research Questions:

Dose female genital mutilation affect sexual relation of married women at Tanta City?

Subjects and method:

Study design:

This study is an exploratory descriptive design.

Setting:

The data were collected from family planning clinics of Tanta University and El-Menshawy Hospitals at Tanta City.

Subjects:

The study included a convenient sample of 80 women from the previously mentioned settings. They were equally divided into 2 groups. The first group consisted of 40 circumcised women, 20 women from each hospital. The second group consisted of 40 non-circumcised women, 20 women from each hospital, based on the following criteria: aged 20 to 45 years old, free from any medical, obstetrical and psychological problems, unprecedented natural childbirth has no more than two children, and living in a nuclear family.

Tools of data collection:

The study used three tools as follows: 
Tool I: A structured questionnaire sheet was developed based on relevant and recent literature. It included the socio demographic characteristics of the women, such as age, education, occupation, and residence.

Tool II:

Clinical observation sheet was developed and used by the researchers to assess the FGM degree: FGC Type I (partial or complete clitoridectomy). Type II (partial clitoridectomy and partial excision of labia minora, Partial clitoridectomy and complete excision of labia minora, complete clitoridectomy and partial excision of labia minora and complete clitoridectomy with complete excision of labia minora). Type III (infibulations). Type IV (pricking, incising, scraping, and cauterization), by whom it was done (professional or non-professional) and assessment of local external female genital deformities included (Clitorial cyst, adhesions between labia majora, adhesions between labia minora, Physical scars around the vaginal opening, narrowing of the vaginal opening and removal of unequal parts on both sides of the vagina). Additionally, the researchers assessed signs of delayed female genital complications such as, keloid, scar abscess, inclusion cyst, urinary tract infection (UTI), and pelvic inflammatory diseases ( PID). The studied women were also asked about the occurrence of immediate complication such as, hemorrhage,fever and dysuria.

Tool III: A modified Arizona Sexual Experiences Scale (ASEX) for assessment female sexual dysfunction. It included 6 items and 36 sub items scored as follows:

Item I: sexual desire/drive included (extremely strong (1), very strong (2), somewhat strong (3), somewhat weak (4), very weak (5), and no sex drive (6).

Item II: arousal included (extremely easy (1), very easily (2), somewhat easily (3), somewhat difficult (4), very difficult (5), and never aroused (6).

Item III: lubrication included (extremely easy (1), very easily (2), somewhat easily (3), somewhat difficult (4), very difficult (5), and never lubricated (6).

Item IV: orgasm included (extremely easy (1), very easily (2), somewhat easily (3), somewhat difficult (4), very difficult (5), and never reach orgasm (6).

Item V: pain during intercourse/dyspareunia included (extremely strong (1), very strong (2), somewhat strong (3), somewhat weak (4), very weak (5), and no pain (6).

Item VI: satisfaction included (extremely satisfying (1), very satisfying (2), somewhat satisfying (3), somewhat unsatisfying (4), very unsatisfying (5), and never satisfied (6).

The scoring system regarding women's sexual dysfunction was as follow: The total score of ASEX was ranged from 6 to 36. According to McGahuey et-al. (1999) women were considered sexually dysfunctioning if they scored more than $60 \%$ (from 21.6 to 36 marks), and if scored on any item 5 or more except dyspare unia, or if scored on any three items 6 marks or more). On the other hand, women were considered sexually functioning if they scored less than $60 \%$ (from 6 to less than 21.6 marks), and if scored on any item 1 to less than 5 or if scored on any three items less than 6 marks).

\section{Method}

1. An official letter was directed from the faculty of Nursing, Tanta Univesity to the head of the Obstetric and Gynecological Medicine Department of both Tanta University, and El-Menshawy Hospital at Tanta in order to obtain their acceptance to collect necessary data from the previous selected settings. The study tools of data collection was developed by the researchers after reviewing the related literaturetranslated and tested for its content validity and reliability by presenting it to five experts in obstetrics and gynecological nursing from the faculty of Nursing. The validity of this tool was calculated by content validity methods and its reliability.

2.Subjects of this study were interviewed during their attendance to family planning clinics at both settings, data collection were accomplished in the morning from 9:00 A.M to 1:00 P.M. two times per week .Over a period of six months (3 months for each hospital) from the beginning of August 2014 to the end of February 2015.

3.Ethical considerations of the study included getting the subjects' consent to participate in the study after reading loudly of the informed consent. Also; confidentiality and privacy were ascertained regarding the collected data.

4.Before embarking on actual study, a pilot study was carried out on eight women attending the both hospitals, 4women from each one (two women from each group). Those women were excluded from the study sample.

Data collection:-

-Tool I: A structured interview questionnaire used by the researchers to assess socio demographic characteristics of each studied woman 
Tool II: (clinical assessment tool) used to assess FGM degree, and the presence of external female genitalia deformity of women during insertion or removal of intrauterine device, or during the vaginal examination by the doctor for any woman complain and asked women about the early and delayed FGM complications.

Tool III: A modified Arizona Sexual Experience Scale (ASEX) assessed women sexual dysfunction.

\section{Statistical analysis:-}

The data were computerized and verified using SPSS (Statistical package for social science) version 18. Analysis of collected data was done through the use of several statistical tests as: Chi-square $\left(\mathrm{x}^{2}\right)$. $\mathrm{P}$ values of $<$ 0.05 were considered statistically significant.

\section{Results}

The collected data was coded, categorized, and analyzed using SPSS.

Table 1: Socio demographic characteristics of the studied subjects $(\mathrm{N}=80)$

\begin{tabular}{|c|c|c|c|c|c|c|c|c|c|}
\hline \multirow{3}{*}{\multicolumn{2}{|c|}{ Socio demographic characteristics }} & \multicolumn{6}{|c|}{ Groups } & \multirow{2}{*}{\multicolumn{2}{|c|}{ Chi-Square }} \\
\hline & & \multicolumn{2}{|c|}{$\begin{array}{c}\text { Group I } \\
(\mathbf{N}=\mathbf{4 0})\end{array}$} & \multicolumn{2}{|c|}{$\begin{array}{l}\text { Group II } \\
(\mathbf{N}=40)\end{array}$} & \multicolumn{2}{|c|}{$\begin{array}{l}\text { Total } \\
(\mathrm{N}=80)\end{array}$} & & \\
\hline & & $\mathbf{N}$ & $\%$ & $\mathbf{N}$ & $\%$ & $\mathbf{N}$ & $\%$ & $\mathrm{X}^{2}$ & P-value \\
\hline \multirow[t]{5}{*}{ Age } & $20-<25$ & 12 & 30.00 & 5 & 12.50 & 17 & 21.25 & \multirow[t]{5}{*}{6.085} & \multirow{5}{*}{0.193} \\
\hline & $25-<30$ & 14 & 35.00 & 12 & 30.00 & 26 & 32.50 & & \\
\hline & $30-<35$ & 4 & 10.00 & 6 & 15.00 & 10 & 12.50 & & \\
\hline & $35-<40$ & 4 & 10.00 & 10 & 25.00 & 14 & 17.50 & & \\
\hline & $40-45$ & 6 & 15.00 & 7 & 17.50 & 13 & 16.25 & & \\
\hline \multirow{4}{*}{$\begin{array}{l}\text { Woman's } \\
\text { education }\end{array}$} & Illiterate\& Elementary & 0 & 0.00 & 2 & 5.00 & 2 & 2.50 & \multirow[t]{4}{*}{6.772} & \multirow[t]{4}{*}{0.238} \\
\hline & Preparatory & 2 & 5.00 & 3 & 7.50 & 5 & 6.25 & & \\
\hline & Secondary & 11 & 27.50 & 6 & 15.00 & 17 & 21.25 & & \\
\hline & University & 23 & 57.50 & 21 & 52.50 & 44 & 55.00 & & \\
\hline \multirow{3}{*}{$\begin{array}{l}\text { Woman's } \\
\text { occupation }\end{array}$} & House wife & 17 & 42.50 & 13 & 32.50 & 30 & 37.50 & \multirow[t]{3}{*}{2.133} & \multirow[t]{3}{*}{0.344} \\
\hline & Non professional work & 20 & 50.00 & 20 & 50.00 & 40 & 50.00 & & \\
\hline & Professional work & 3 & 7.50 & 7 & 17.50 & 10 & 12.50 & & \\
\hline \multirow[t]{2}{*}{ Residency } & Rural & 9 & 22.50 & 27 & 67.50 & 58 & 72.50 & \multirow[t]{2}{*}{1.003} & \multirow[t]{2}{*}{0.317} \\
\hline & Urban & 31 & 77.50 & 13 & 32.50 & 22 & 27.50 & & \\
\hline
\end{tabular}

\section{Group I= Non - circumcised women.}

\section{Group II= Circumcised women.}

Table 1: shows the socio demographic data of the studied women. It was found that among the non circumcised and circumcised studied women $(35 \% \& 30 \%$ respectively) had $25-<30$ years old with non significant differences. There were no significant differences between both groups regarding their education level, occupation and residence. The table also shows that nearly three quarters of circumcised women( $72.50 \%)$ from rural areas.

Table 2: Type and performance of Female genital mutilation (FGM) of the studied women $(\mathrm{N}=40)$

\begin{tabular}{||l|l|l|l||}
\hline \multicolumn{3}{|c|}{ Groups of Female genital cutting } \\
\hline Type and performance of Female genital cutting (N= 40) & $\mathbf{N}$ & $\mathbf{\%}$ \\
\hline \multirow{2}{*}{ Type I } & Partial clitoridectomy & 9 & 22.5 \\
\cline { 2 - 4 } & Complete clitoridectomy & 4 & 10.0 \\
\hline \multirow{3}{*}{ Total } & & $\mathbf{N}$ & $\mathbf{\%}$ \\
\cline { 3 - 4 } & & 13 & 32.50 \\
\hline \multirow{4}{*}{ Type II } & Partial clitoridectomy and partial excision of labia minora & 6 & 15.0 \\
\cline { 2 - 4 } & Partial clitoridectomy and complete excision of labia minora & 3 & 7.5 \\
\cline { 2 - 4 } & Complete clitoridectomy and partial excision of labia minora & 6 & 15.0 \\
\cline { 2 - 4 } & Complete clitoridectomy and complete excision of labia minor & 12 & 30.0 \\
\hline \multirow{2}{*}{ Total } & & $\mathbf{N}$ & $\mathbf{\%}$ \\
\hline \multirow{2}{*}{ FGM performance } & By professional health care provider & 27 & 67.50 \\
\cline { 2 - 4 } & By non-professional practitioners & 10 & 25.0 \\
\hline
\end{tabular}

Table 2: shows the degree/Type of Female genital cutting of the studied women. It shows that $32.50 \%$ among circumcised studied women had female genital cutting type I and 67,50\% of them had female genital cutting type II. The table also shows that $75 \%$ of the studied women performed their circumcision by non-professional person. 
Table 3: Immediate and delayed complications and local deformities related to female genital cutting( $\mathrm{N}=40)$

\begin{tabular}{|c|c|c|c|}
\hline \multicolumn{2}{|c|}{ Immediate complications and local deformities of female genital cutting } & $\mathbf{N}$ & $\%$ \\
\hline \multirow{8}{*}{$\begin{array}{l}\text { Immediate and delayed complications } \\
\text { of female genital cutting } \\
\text { (N didn't add to } 40 \text { as there were more } \\
\text { than one answer) }\end{array}$} & Hemorrhage & 7 & 17.50 \\
\hline & Fever & 13 & 32.50 \\
\hline & Pain & 13 & 32.50 \\
\hline & Urinary retention & 7 & 17.50 \\
\hline & Recurrent UTI & 6 & 15.00 \\
\hline & Dyspareunia & 18 & 45.00 \\
\hline & Dysmenorrhea & 22 & 55.00 \\
\hline & Dystocia & 6 & 15.00 \\
\hline \multirow{2}{*}{$\begin{array}{l}\text { Presence of external female genitalia } \\
\text { deformity }\end{array}$} & Yes & 22 & 55.00 \\
\hline & No & 18 & 45.00 \\
\hline \multirow{6}{*}{$\begin{array}{l}\text { Types of deformity present (all that } \\
\text { apply) }(\mathrm{N}=22)\end{array}$} & Clitorial cyst & 3 & 13.64 \\
\hline & Adhesions between labia minora & 4 & 18.18 \\
\hline & $\begin{array}{l}\text { Physical scars around the vaginal } \\
\text { opening }\end{array}$ & 6 & 27.27 \\
\hline & Narrowing of the vaginal opening & 2 & 9.09 \\
\hline & $\begin{array}{l}\text { Removal of unequal parts on both } \\
\text { sides of the vagina }\end{array}$ & 5 & 22.73 \\
\hline & $\begin{array}{l}\text { Leave skin grow and hanging after } \\
\text { FGC }\end{array}$ & 2 & 9.09 \\
\hline
\end{tabular}

Table 3: Shows the immediate and delayed complications and the Presence of external female genitalia deformity. It shows that nearly the third $32.50 \%$ among circumcised studied women had fever and dysurea and nearly the fifth $(17.50 \%)$ of them had hemorrhage as immediate complications and $(45 \%$ and $55 \%$ respectively of them) had dyspareunia and Dysmenorrhea as delayed complications. The table also shows that $55 \%$ of the circumcised studied women had external female genitalia deformity.

Table 4: Sexual dysfunction among non-circumcised and circumcised women

\begin{tabular}{|c|c|c|c|c|c|c|c|c|c|}
\hline \multirow{3}{*}{\multicolumn{2}{|c|}{ Sexual dysfunction }} & \multicolumn{6}{|c|}{ Groups } & \multirow{2}{*}{\multicolumn{2}{|c|}{ Chi-Square }} \\
\hline & & \multicolumn{2}{|c|}{$\begin{array}{c}\text { Group I } \\
(\mathrm{N}=40)\end{array}$} & \multicolumn{2}{|c|}{$\begin{array}{c}\text { Group II } \\
(\mathrm{N}=\mathbf{4 0})\end{array}$} & \multicolumn{2}{|c|}{$\begin{array}{l}\text { Total } \\
(\mathrm{N}=80)\end{array}$} & & \\
\hline & & $\mathbf{N}$ & $\%$ & $\mathbf{N}$ & $\%$ & $\mathbf{N}$ & $\%$ & $\mathbf{X}^{2}$ & P-value \\
\hline \multirow[t]{6}{*}{ Desire / drive } & Extremely strong & 5 & 12.50 & 1 & 2.50 & 6 & 7.50 & \multirow[t]{5}{*}{25.692} & \multirow[t]{5}{*}{$<0.001 *$} \\
\hline & Very strong & 18 & 45.00 & 5 & 12.50 & 23 & 28.75 & & \\
\hline & Somewhat strong & 15 & 37.50 & 17 & 42.50 & 32 & 40.00 & & \\
\hline & Somewhat weak & 2 & 5.00 & 12 & 30.00 & 14 & 17.50 & & \\
\hline & Very weak & 0 & 0.00 & 5 & 12.50 & 5 & 6.25 & & \\
\hline & No sex drive & 0 & 0 & 0 & 0 & 0 & 0 & & \\
\hline \multirow[t]{6}{*}{ Arousal } & Extremely easy & 4 & 10.00 & 0 & 0.00 & 4 & 5.00 & \multirow[t]{5}{*}{31.965} & \multirow[t]{5}{*}{$<0.001 *$} \\
\hline & Very easily & 23 & 57.50 & 8 & 20.00 & 31 & 38.75 & & \\
\hline & Somewhat easily & 11 & 27.50 & 13 & 32.50 & 24 & 30.00 & & \\
\hline & Some what difficult & 2 & 5.00 & 9 & 22.50 & 11 & 13.75 & & \\
\hline & Very difficult & 0 & 0.00 & 10 & 25.00 & 10 & 12.50 & & \\
\hline & Never aroused & 0 & 0 & 0 & 0 & 0 & 0 & & \\
\hline \multirow[t]{6}{*}{ Lubrication } & Extremely easy & 2 & 5.00 & 1 & 2.50 & 3 & 3.75 & \multirow[t]{6}{*}{26.719} & \multirow[t]{6}{*}{$<0.001 *$} \\
\hline & Very easily & 22 & 55.00 & 7 & 17.50 & 29 & 36.25 & & \\
\hline & Somewhat easily & 12 & 30.00 & 10 & 25.00 & 22 & 27.50 & & \\
\hline & Some what difficult & 4 & 10.00 & 12 & 30.00 & 16 & 20.00 & & \\
\hline & Very difficult & 0 & 0.00 & 9 & 22.50 & 9 & 11.25 & & \\
\hline & Never & 0 & 0.00 & 1 & 2.50 & 1 & 1.25 & & \\
\hline \multirow[t]{6}{*}{ Orgasm } & Extremely easy & 2 & 5.00 & 1 & 2.50 & 3 & 3.75 & \multirow[t]{6}{*}{27.778} & \multirow[t]{6}{*}{$<0.001 *$} \\
\hline & Very easily & 20 & 50.00 & 6 & 15.00 & 26 & 32.50 & & \\
\hline & Somewhat easily & 12 & 30.00 & 12 & 30.00 & 24 & 30.00 & & \\
\hline & Some what difficult & 6 & 15.00 & 7 & 17.50 & 13 & 16.25 & & \\
\hline & Very difficult & 0 & 0.00 & 11 & 27.50 & 11 & 13.75 & & \\
\hline & Never reach orgasm & 0 & 0.00 & 3 & 7.50 & 3 & 3.75 & & \\
\hline \multirow[t]{5}{*}{ Dyspareunia } & Extremely strong & 0 & 0.00 & 9 & 22.50 & 9 & 11.25 & \multirow[t]{5}{*}{21.525} & \multirow[t]{5}{*}{$<0.001 *$} \\
\hline & Some what strong & 3 & 7.50 & 9 & 22.50 & 12 & 15.00 & & \\
\hline & Some what weak & 11 & 27.50 & 8 & 20.00 & 19 & 23.75 & & \\
\hline & Very weak & 15 & 37.50 & 5 & 12.50 & 20 & 25.00 & & \\
\hline & No pain & 11 & 27.50 & 9 & 22.50 & 20 & 25.00 & & \\
\hline \multirow[t]{6}{*}{ Satisfaction } & Extremely satisfying & 4 & 10.00 & 0 & 0.00 & 4 & 5.00 & \multirow[t]{6}{*}{30.829} & \multirow[t]{6}{*}{$<0.001 *$} \\
\hline & Very satisfying & 18 & 45.00 & 8 & 20.00 & 26 & 32.50 & & \\
\hline & Some what satisfying & 13 & 32.50 & 14 & 35.00 & 27 & 33.75 & & \\
\hline & Some what unsatisfying & 5 & 12.50 & 3 & 7.50 & 8 & 10.00 & & \\
\hline & Very unsatisfying & 0 & 0.00 & 4 & 10.00 & 4 & 5.00 & & \\
\hline & Never satisfied & 0 & 0.00 & 11 & 27.50 & 11 & 13.75 & & \\
\hline
\end{tabular}




\section{Group I= Non- circumcised women.}

\section{Group II= Circumcised women.}

Table 4: shows Sexual dysfunction reported by the studied non-circumcised and circumcised women. The table represents that the differences was statistically significant between the group I and group II regarding sexual desire, arousal, lubrication, orgasm, Dyspareunia and sexual dysfunction $\left(\mathrm{P}<<0.001 *,<0.001^{*},<0.001 *\right.$, $<0.001 * \&<0.001 *$ respectively)

Table 5: Total sexual functional and dysfunctional among non-circumcised and circumcised women.

\begin{tabular}{|c|c|c|c|c|}
\hline \multirow{2}{*}{ ASEX score } & \multicolumn{4}{|c|}{ Groups } \\
\hline & $\mathbf{N}-\%$ & Group I & Group II & Total \\
\hline \multirow{2}{*}{ Sexual functional } & $\mathrm{N}$ & 6 & 1 & 7 \\
\hline & $\%$ & 15.00 & 2.50 & 8.75 \\
\hline \multirow[t]{2}{*}{ Sexual dysfunctional } & $\mathrm{N}$ & 34 & 39 & 73 \\
\hline & $\%$ & 85.00 & 97.50 & 91.25 \\
\hline \multirow[t]{2}{*}{ Total } & $\mathrm{N}$ & 40 & 40 & 80 \\
\hline & $\%$ & 100.00 & 100.00 & 100.00 \\
\hline \multirow[t]{2}{*}{ Chi-square } & $\mathbf{X}^{2}$ & 4.305 & & \\
\hline & P-value & $0.038^{*}$ & & \\
\hline
\end{tabular}

Group I= Non - circumcised women.

\section{Group II= Circumcised women.}

Table 5: shows the sexual functional and dysfunctional among circumcised and non- circumcised women .It shows that sexual dysfunction was significantly higher among circumcised women compared to noncircumcised ones $(97.5 \% \& 85 \%)$ respectively $\left(\mathrm{P}=0.038^{*}\right)$

Figure 1: Relationship between sexual functional and the female genital mutilation type 1.

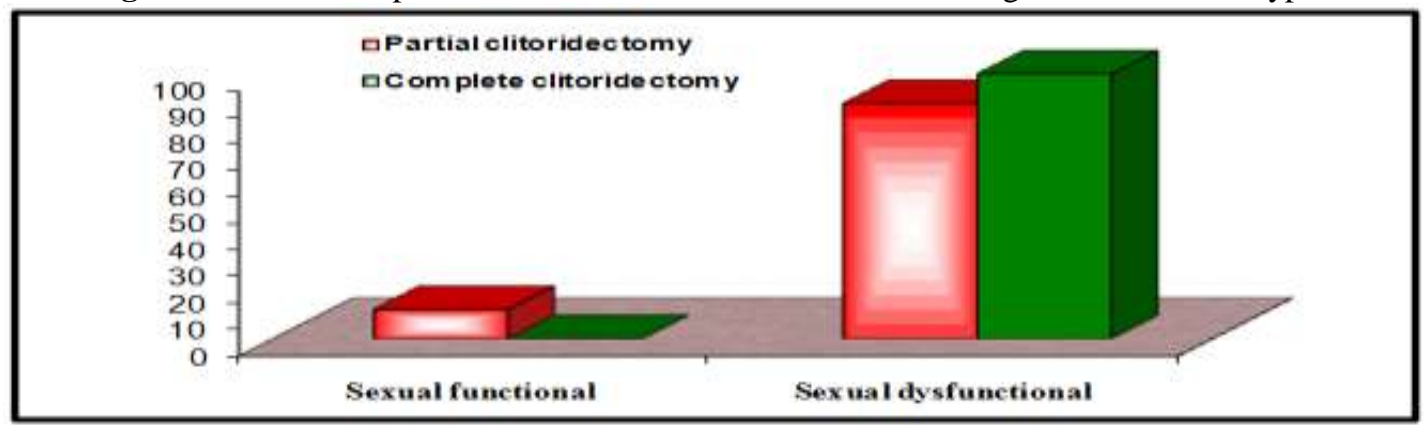

Figure 1: shows the effect of female genital mutilation types I and sexual functional .It shows that the majority of the studied women who have Partial clitoridectomy $88.89 \%$ had sexual dysfunctional. The table also shows that all the studied women who have complete clitoridectomy $100 \%$ had sexual dysfunctional.

Table 6: Relationship between Socio-demographic characteristics of the studied non- circumcised women and sexual function

\begin{tabular}{|c|c|c|c|c|c|c|c|}
\hline \multicolumn{2}{|c|}{ Non Circumcised woman } & \multirow[t]{2}{*}{$\mathbf{N}$} & \multicolumn{3}{|c|}{ ASEX score } & \multicolumn{2}{|c|}{ ANOVA or T-test } \\
\hline & & & Mean & \pm & SD & F or $T$ & P-value \\
\hline \multirow[t]{5}{*}{ Age } & $<25$ & 12 & 29.583 & \pm & 4.582 & \multirow[t]{5}{*}{8.339} & \multirow[t]{5}{*}{$0.000^{*}$} \\
\hline & $25-<30$ & 14 & 26.786 & \pm & 4.318 & & \\
\hline & $30-<35$ & 4 & 29.500 & \pm & 2.380 & & \\
\hline & $35-<40$ & 4 & 24.000 & \pm & 6.976 & & \\
\hline & $40-45$ & 6 & 24.333 & \pm & 4.676 & & \\
\hline \multirow[t]{4}{*}{ Woman's education } & Illiterate \& Elementary & 4 & 20.500 & \pm & 3.000 & \multirow[t]{4}{*}{8.339} & \multirow[t]{4}{*}{$0.000^{*}$} \\
\hline & Preparatory & 2 & 19.000 & \pm & 0.000 & & \\
\hline & Secondary & 11 & 27.909 & \pm & 4.867 & & \\
\hline & University & 23 & 28.826 & \pm & 3.589 & & \\
\hline \multirow[t]{3}{*}{ Woman's occupation: } & House wife & 3 & 28.000 & \pm & 1.732 & \multirow[t]{3}{*}{0.137} & \multirow[t]{3}{*}{0.872} \\
\hline & Non professional work & 17 & 27.588 & \pm & 4.583 & & \\
\hline & Professional work & 20 & 26.850 & \pm & 5.518 & & \\
\hline \multirow[t]{2}{*}{ Residency } & rural & 9 & 25.000 & \pm & 6.285 & \multirow[t]{2}{*}{-1.301} & \multirow[t]{2}{*}{0.222} \\
\hline & Urban & 31 & 27.903 & \pm & 4.292 & & \\
\hline
\end{tabular}

Table 6: revealed that woman's age and education, were statistically significant factors ( $F=8.339 \& \mathrm{p}=0.000$, $\mathrm{F}=8.339 \& \mathrm{p}=0.000$ respectively) affecting sexual function among non- circumcised women, while women occupation and residency were not statistically significant factors ( $\mathrm{p}=0.872 \& \mathrm{p}=0.22$ respectively). 
Table 7: Relationship between socio-demographic characteristics of studied circumcised women and sexual function

\begin{tabular}{|c|c|c|c|c|c|c|c|}
\hline \multicolumn{2}{|l|}{ Circumcised woman } & \multirow{3}{*}{\begin{tabular}{|l}
$\mathbf{N}$ \\
5
\end{tabular}} & \multicolumn{3}{|c|}{ ASEX score } & \multicolumn{2}{|c|}{ ANOVA or T-test } \\
\hline & & & Mean & \pm & SD & F or $\mathbf{T}$ & P-value \\
\hline \multirow[t]{5}{*}{ Age } & $<25$ & & 26.800 & \pm & 2.588 & \multirow[t]{5}{*}{0.839} & \multirow[t]{5}{*}{0.532} \\
\hline & $25-<30$ & 12 & 25.833 & \pm & 2.552 & & \\
\hline & $30-<35$ & 6 & 25.000 & \pm & 4.243 & & \\
\hline & $35-<40$ & 10 & 26.900 & \pm & 2.726 & & \\
\hline & $40-45$ & 7 & 25.000 & \pm & 1.155 & & \\
\hline \multirow[t]{4}{*}{ Woman's education } & Illiterate \& Elementary & 2 & 27.500 & \pm & 0.707 & \multirow[t]{4}{*}{0.839} & \multirow[t]{4}{*}{0.532} \\
\hline & Preparatory & 3 & 25.667 & \pm & 2.082 & & \\
\hline & Secondary & 6 & 26.167 & \pm & 2.787 & & \\
\hline & University & 21 & 26.143 & \pm & 3.087 & & \\
\hline \multirow[t]{3}{*}{ Woman's occupation } & House wife & 13 & 27.231 & \pm & 2.279 & \multirow[t]{3}{*}{4.966} & \multirow[t]{3}{*}{$0.012^{*}$} \\
\hline & Non professional work & 20 & 25.950 & \pm & 2.417 & & \\
\hline & Professional work & 7 & 23.571 & \pm & 2.992 & & \\
\hline \multirow[t]{2}{*}{ Residency } & Rural & 27 & 26.000 & \pm & 2.614 & \multirow[t]{2}{*}{0.080} & \multirow[t]{2}{*}{0.937} \\
\hline & Urban & 13 & 25.926 & \pm & 2.814 & & \\
\hline
\end{tabular}

Table 7: revealed that from all socio -demographic factors, only the woman's occupation was a significant factor affecting sexual function among circumcised women $(\mathrm{F}=4.966 \& \mathrm{p}=0.012)$.

Table 8: Women's perception of the role of Obstetrical and Gynecological nurses in relation to sexual dysfunction and female genital mutilation

\begin{tabular}{|c|c|c|c|c|c|c|c|c|}
\hline \multirow{3}{*}{$\begin{array}{l}\text { Role of Obstetrical and Gynecological } \\
\text { nurse in relation to female genital } \\
\text { mutilation and sexual dysfunction }\end{array}$} & \multicolumn{6}{|c|}{ Groups } & \multirow{2}{*}{\multicolumn{2}{|c|}{ Chi-Square }} \\
\hline & \multicolumn{2}{|c|}{$\begin{array}{l}\text { Group I } \\
(\mathrm{N}=40)\end{array}$} & \multicolumn{2}{|c|}{$\begin{array}{l}\text { Group II } \\
(\mathrm{N}=\mathbf{4 0})\end{array}$} & \multicolumn{2}{|c|}{$\begin{array}{l}\text { Total } \\
(\mathrm{N}=\mathbf{8 0})\end{array}$} & & \\
\hline & $\mathbf{N}$ & $\%$ & $\mathbf{N}$ & $\%$ & $\mathbf{N}$ & $\%$ & $\mathbf{X}^{2}$ & P-value \\
\hline Health education to women & 30 & 75.00 & 31 & 77.50 & 61 & 76.25 & 0.069 & 0.793 \\
\hline Health education to the community & 25 & 62.50 & 25 & 62.50 & 50 & 62.50 & 0.000 & 1.000 \\
\hline Referral to social support & 0 & 0.00 & 3 & 7.50 & 3 & 3.75 & 4.276 & $0.039 *$ \\
\hline Implementation of health care laws & 15 & 37.50 & 21 & 52.50 & 36 & 45.00 & 1.818 & 0.178 \\
\hline Call for further action of prevention & 1 & 2.50 & 8 & 20.00 & 9 & 11.25 & 6.889 & $0.009^{*}$ \\
\hline
\end{tabular}

\section{Group I= Non -circumcised women.}

Group II= Circumcised women.

Table (8): shows women's perception of the role of obstetrical and gynecological nurses in relation to sexual dysfunction and female genital mutilation. The table shows that the highest percents of group I \& group II (75\% $\$ 77.50 \%$ respectively) needed for health education to women. $37.50 \%$ of group I and $52,50 \%$ of group II encourage the Implementation of health care laws. The table represents that the differences was statistically significant between the group I and group II regarding the referral to social support and Call for further action of prevention $(\mathrm{P}<0.039 *,<0.009 *$ respectively)

\section{Discussion}

Female genital mutilation (FGM) is a harmful traditional practice rooted in religious, personal and societal beliefs to control women's sexuality. This practice violates and abuses women's rights to maintain the quality of their life ${ }^{(18)}$. Thus, the present study aimed to investigate the effect of FGM on sexual relation among married women at Tanta city. The present study shows that there were no statistically significant differences between circumcised and non circumcised women regarding their socioeconomic data (women age, woman's education, occupation, and residency). These findings are in accordance with the findings of previous critical and comparative analysis that investigated female genital mutilation in the Demographic and Health Surveys done by Yoder et-al (2004). The results indicated that FGM is widespread among the surveyed countries including Egypt, Eritrea, Mali, Sudan, Yemen, as well as immigrants of Europe, and regions in northern and South America. It occurs in all groups, especially Muslim women despite the fact that the practice predates Islam. ${ }^{(19)}$

Regarding the type of FGM, the present study shows that nearly one third of women had genital mutilation type I and more than two third of them had female genital mutilation type II. This result is in accordance with WHO (2006) reported that in Africa, the most common type of FGC is type II which accounts for up to $80 \%$ of all cases ${ }^{(20)}$. On the other hand, this result disagrees with Al-Hussaini TK(2003) and Anis TH et-al. (2012) who found that the most common form of FGC still widely practiced throughout Egypt is type $\mathrm{I}^{(21,17)}$. This disagreement may be due to the fact that nearly one quarter of the studied women in the present study were from rural origins and three quarters of them performed FGM by non-professional practitioners. These results run against the results of Tag-Eldin $\mathbf{M}$ et al. (2008) who found that physicians in their clinics 
performed $49.7 \%$ of FGC and $7.6 \%$, at their hospitals while, nurses performed only $10.4 \%$. Dayas and midwives performed $29.3 \%$ of FGC while lay persons ( non-professional) performed only $3 \%{ }^{(22)}$.

Regarding the immediate and delayed complications of FGM, this study shows that nearly one third of circumcised studied women had fever and pain, from the researchers point of view this is may be due to that FGM occurred by follow septic technique. Less than one fifth had hemorrhage because most of the procedure of FGM was done by non-professional and nearly one half had dyspareunia. This is may be due to that the scar tissue from FGM led to dyspareunia, lack of sex interest, vaginal dryness, and lower sexual satisfaction. These results correspond with Tag-Eldin $M$ et al. (2008), Elgaali $M$ et al.(2005), Almroth $L$ et al.(2005), and Hakim LY et al(2001) who stated that the early and late complications of FGC were severe pain, bleeding, incontinence, infections, sexual problems, primary infertility and difficult labor with high episiotomy rate ${ }^{(22-25)}$. The present study shows that more than the half of circumcised studied women had external female genitalia deformity. The result of the present study is accepted because FGM comprises various procedures which remove or damage the external female genital organs for no medical reason. Also genital scarring is common but can be very variable due to the extent of tissue removed and immediate complication such as infection ${ }^{(26)}$.

Regarding sexual dysfunction among non- circumcised and circumcised women, the study represents that the differences was statistically highly significant between non-circumcised and circumcised women regarding sexual desire, arousal, lubrication, orgasm, dyspareunia and sexual satisfaction. Furthermore, the majority of the circumcised women who have partial clitoridectomy and all the women who have complete clitoridectomy had sexual dysfunctional. Although studies on sexual function have also been small, this result is compatible with Rigmor C et-al. (2012) who examined FGM/C effect on women's sexual functioning. They found that women with FGM/ were likely not to have sexual desire, dyspareunia and reduced sexual satisfaction twice more than women without FGM ${ }^{(27)}$. As regarding to the total score of sexual dysfunction, the present study represented that sexual dysfunction was significantly higher among circumcised women compared to non-circumcised ones .This result agree with Anis TH et-al.(2012) who represented that non - circumcised women had significantly higher total score of sexual functioning compared with circumcised women regarding sexual desire, arousal, lubrication, orgasm, and satisfaction domains ${ }^{(17)}$. It is obvious that FGC does not eliminate sexual pleasure for all women who underwent partial clitoridectomy, and the effect of FGM on a woman's sexual experience varies depending on many other factors. This can be explained as sexual excitement for a woman during intercourse involves a complex series of nerve endings being activated and stimulated around her vagina, vulva (labia minora and majora) and clitoris, psychological response and mind-set is also important ${ }^{(28,29)}$.

The present study revealed that woman's age and education were statistically significant factors affecting sexual function among non- circumcised women while the women's occupation and residency were not statistically significant factors. It is obvious from this results that despite female genital mutilation, there are other factors which can affect women sexual function such as, age and educational level which has effect and impact on the individual's personalities and their abilities to ask for help regarding any problem facing them, including sexual relation. This result match up with Hayes R \& Dennerstein L (2005 who studied the impact of aging on sexual function and sexual dysfunction among women, they found that a woman's sexual function declines with age. Specifically, desire, frequency of orgasm, and frequency of sexual intercourse ${ }^{(30)}$. Also the result conform with Çayan S et al. (2004) who represents that the prevalence of female sexual dysfunction including desire, arousal, lubrication, orgasm, satisfaction and pain problems increases with age. In addition, the presence of a lower educational level and unemployment status are important risk factors that may cause sexual dysfunction ${ }^{(31)}$.

The present study revealed that from all socio - demographic factors, only the woman's occupation was a significant factor affecting sexual function among circumcised women. This result was supported by Ramezani TF et al. Who found that new tasks in women's' life such as their occupation or engagement in care of their children were affect women sexual function From the researchers point of view this results was accepted because the women occupation and their more interaction with the community was affect their sexual function instead of their circumcision ${ }^{(32)}$.

Regarding the role of Obstetrical and Gynecological nurses in relation to sexual dysfunction and female genital cutting, the present study shows that the studied women in dire need of health education ,implementation of health care laws, referral to social support and Call for further action of FGM . From the researcher point of view the result of the present study was acceptable and predictable because there is no doubt that the level of university education among more than the half of studied women has helped them to increase their awareness regarding the negative impact of FGM on women health status physically and psycho-sexually. This result correspond with the study done by Gage and Rossem (2006) to examine the relationship of socioeconomic 
factors and gender to attitudinal support for the discontinuation of FGC among men and women aged 15-59 in Guinea showed that attitudinal support for FGC discontinuation was more prevalent among men than women. The chances of supporting the discontinuation of FGC were negatively related to beliefs in social approval of and religious support and its enhancement of women's marriage ability ${ }^{(33)}$.Communities express their desire to eradicate the practice, religious institutions are indicating that FGC is not a requirement, and governments are approving programs that educate the nation about the harms of FGM ${ }^{(34)}$.

\section{Recommendation}

This study recommended that there is a crucial need for focusing on more orientation of the community regarding the effect and the hazard of FGM on sexual relation and its role on occurrence of sexual dysfunction.

\section{References}

[1]. World Health Organization (WHO), Female Genital Mutilation, fact sheet updated February 2016, retrieved from http://www.who.int/mediacentre/factsheets/fs241/en.

[2]. WHO. Female genital mutilation/cutting: a global concern. Geneva: UNICEF; 2016,

retrievedfrom,http://data.unicef.org/resources/female-genital-mutilation-cutting-a-global-concern.html, accessed 26 April 2016.

[3]. WHO. Classification of female genital mutilation, sexual and reproductive health http://www.who.int/reproductivehealth/topics/fgm/overview/en,October,2016.

[4]. WHO/UNICEF UNFPA. Female Genital Mutilation. A joint statement. World Health Organization, Geneva, May 2014.

[5]. United Nations Children's Fund, Female Genital Mutilation/Cutting: A global concern, UNICEF, New York, 2016.

[6]. The State of the World's Children 2015: Executive Summary, New York: UNICEF, November 2014, Table 9, pp. 84-9.

[7]. Demographic and Health Surveys, accessed online at www.measuredhs.com, on July 10, 2007.

[8]. Sami A., and Abu-Sahlieh A. Female circumcision. Religious arguments about male and female circumcision, 2003. Available at April 6, 2015.

[9]. Berg RC., and Denison E. A Tradition in Transition: Factors Perpetuating and Hindering the Continuance of Female Genital Mutilation Cutting (FGM/C) Summarized in a Systematic Review .Health Care for Women International, 2013;34(10): 837-95.

[10]. Egypt Bans Female Circumcision. MSNBC News, accessed online at http://www.msnbc.msn.com. Available at July 12, 2016.

[11]. El-Saadawi N. The hidden face of Every Women in the Arab World, translated and edited by Sherif Hetata, Zed Press, London, 1980 .

[12]. WHO. Female genital cutting, 2006. Available at Mar 24, 2016. Retrieved from: http//ww.who.int/mediacentre/factsheets /fs241/en/index.html.

[13]. Nour N. Female circumcision and genital cutting. 2009. Available at Jun 4,2010. Retrieved from http://www.uptodate.com/patients/content/topic.

[14]. Nour N. Urinary calculus associated with female genital cutting. Journal Obstet Gynecol Res ,2006; $107: 521$

[15]. Fernandez S., and Noel J. Neuroma of the clitoris after female genital cutting. Journal Obstet Gynecol Res, 2003; $101: 1053$.

[16]. WHO. Female genital cutting exposes women and babies to significant risk at child birth. Geneva. 2 June, 2006.

[17]. Anis TH., Aboul Gheit S., Awad HH., Saied HS. Effects of female genital cutting on the sexual function of Egyptian women. A cross- sectional study. Journal Sex Med. 2012;9(10):2682-92.

[18]. Vissandjée B, Denetto S, Migliardi P and Proctor J. Female genital cutting (FGC) and the ethics of care: community engagement and cultural sensitivity at the interface of migration experiences. licensee BioMed Central Ltd. 2014BMC International Health and Human Rights2014 14:13

[19]. Yoder PS., Abderrahim N., and Zhuzhuni A. Female genital cutting in the Demographic and Health Surveys: a critical and comparative analysis. Calverton, Maryland, ORC Macro, 2004;76 (7).

[20]. Female genital mutilation [Fact sheet no. 241]. Geneva: WHO; 2006.

[21]. Al-Hussaini TK. Female genital cutting - types, motives and perineal damage in laboring Egyptian women. Med Pinc Pract 2003;12(2):123-8

[22]. Tag-Eldin M., Gadallah M., Al-Tayeb M., Abdel-Aty M., Mansour E., Sallem M., Prevalence of female genital cutting among Egyptian girls Bulletin of the World Health Organization, past issues. 2008; 86(4): 241-320.

[23]. Elgaali M., Strevens H., and Mardh PA. Female genital mutilation an exported medical hazard. Eur J Contracept Reprod Health Care 2005; 10(2): 93-7.

[24]. Almroth L., Elmusharaf S., El Hadi N., Obeid A., El Sheikh MA., and Elfadil SM. Primary infertility after genital mutilation in girlhood in Sudan: a case-control study. Lancet 2005; 366(9483): 385-91.

[25]. Hakim LY. Impact of female genital mutilation on maternal and neonatal outcomes during parturition. East Afr Med Journal 2001; 78(5): 255-8.

[26]. Reisel D and Creightom SM.Long term health consequences of Female Genital Mutilation., US National Library of Medicine. National Institutes of Health

[27]. Rigmor C., and Denison E. Does Female Genital Mutilation/Cutting (FGM/C) Affect Women's Sexual Functioning? A Systematic Review of the Sexual Consequences of FGM/C Journal of Sexuality Research and Social Policy March 2012;9(1): 41-56. 2015 Jan;80(1):48-51 (PubMmed)

[28]. Komisaruk B., et al. The Science of Orgasm. JHU Press, For an interview with two of the researchers, see "Exploring the MindBody Orgasm", 2006 Retrieved from http://www.wired.com/medtech/health/news/2007/01/72325.

[29]. Mah K., and Binik YM. "Are orgasms in the mind of the body? Psychosocial versus physiological correlates of orgasmic pleasure and satisfaction". Journal of Sex and Marital Therapy 2005;31:187-200.

[30]. Hayes R., and Dennerstein L. The impact of aging on sexual function and sexual dysfunction in women: a review of population based studies. Sex Med. 2005 May; 2(3):317-30

[31]. Çayan S., Akbay E., Bozlu M., Canpolat B., Acar D., and Ulusoy E. The Prevalence of Female Sexual Dysfunction and Potential Risk Factors That May Impair Sexual Function in Turkish Women. Urologia journal2004;72(1):52-7.Original Paper KARGER medical and scientific publisher.

[32]. Ramezani TF, Farahmand M, Mehrabi Y, Malekafzali H, Abedini M. Prevalence Of Female Sexual Dysfunction and its Correlated Factors: A Population Based Study. Payesh. 2012;11(6):869-875.

[33]. Gage AJ., and Van Rossem R. Attitudes toward the discontinuation of female genital cutting among men and women in Guinea. nt J Gynaecol Obstet. 2006 Jan;92(1):92-6

[34]. Boddy J. The normal and the aberrant in female genital cutting. Journal of Ethnographic theory.vol 6, No2 (2016). 